\title{
Performance assessment of amplification and discrimination electronic devices for passive neutron measurements
}

\author{
Ben Mosbah M. ${ }^{1 *}$, Eleon C. ${ }^{1}$, Passard C. ${ }^{1}$, Loridon J. ${ }^{1}$, Perot B. ${ }^{1}$, Barbot L. ${ }^{2}$ and Grassi G. ${ }^{3}$
}

\begin{abstract}
The knowledge of the fissile material mass is a key challenge to enhance radioactive waste management and to ensure a high level of safety in nuclear industry. Data is analyzed according to the principles of the neutron measurement techniques. As proportional counters filled with ${ }^{3} \mathrm{He}$ gas display high neutron detection efficiency and a good gamma-ray discrimination, they are the reference detector for passive neutron coincidence counting. A charge preamplifier or a current amplifier, depending on applications, collects the electric pulse produced by neutron interaction in the ${ }^{3} \mathrm{He}$ gas and a threshold discriminator produces a logic pulse used for neutron counting. This paper describes the performance assessment of different commercially available electronics from Mirion Technologies, Precision Data Technology (PDT), Mesytec, as well as MONACO electronics originally developed by CEA LIST for fission chamber measurements in experimental reactors. Comparative passive neutron measurements are carried out with these electronics at CEA/DEN Nuclear Measurement Laboratory in Cadarache. Overall, PDT and Mesytec electronics show similar detection efficiency as the ACH-NA98 charge amplifier, which is commonly used in our laboratory for such applications. However, MONACO electronics have a lower detection efficiency, similar to Mirion 7820 current amplifier used in specific high-count rate applications. An optimisation of MONACO settings would probably be necessary to adapt to ${ }^{3} \mathrm{He}$ counters instead of fission chambers.
\end{abstract}

\section{INTRODUCTION}

The knowledge of the fissile material mass is a key challenge to enhance radioactive waste management and to ensure a high level of safety in nuclear industry.

Proportional counters filled with ${ }^{3} \mathrm{He}$ gas display high neutron detection efficiency and good gamma-ray discrimination. Therefore, they are an obvious choice for neutron detection, especially for radioactive waste characterization [1]. An amplifier and a threshold discriminator transform the analog pulse of the counter into a logic signal for neutron counting purpose. The amplification and discrimination electronics is essential to ensure linear counting with respect to the quantity of plutonium, especially to avoid count losses due to dead time and pile-up [2].

This paper describes an experimental benchmark of commercially available electronics. The 7820 current amplifier and the ACH-NA98 charge amplifier from Miron
Technologies, which have been widely qualified at the Nuclear Measurement Laboratory (LMN) of CEA/DEN in Cadarache , are used as reference electronics. The ACH-NA98 module allows the best performances in terms of detection efficiency, while 7820 amplifier is more robust in case of high-count rate and high gamma radiation level. The PDT10A and PDT10M+PDT12S modules from Precision Data Technology, as well as MRS2000-1 and MRS2000-2 amplifiers from Mesytec, have been compar]ed to our reference electronics. CEA's prototype amplifier MONACO is also studied: this device was originally designed for fission chamber measurements in experimental reactors [3]. All tested electronics are plugged to the same ${ }^{3} \mathrm{He}$ detectors to compare their performances.

The experimental programme has been carried out at the LMN. The first section describes the experimental setup, the detection system, and the electronics. Experiments are presented in the second section. The detector efficiency and dieaway time are determined for each configuration.

\section{EXPERIMENTAL SETUP}

Two polyethylene detection blocks wrapped with a $2 \mathrm{~mm}$ cadmium envelop and covered with a $5 \mathrm{~mm} \mathrm{~B} \mathrm{~B}_{4} \mathrm{C}$ plug are used (see Fig. 1 and 2). They can host up to seven $65 \mathrm{NH} 45{ }^{3} \mathrm{He}$ proportional counters from Canberra (currently Mirion Technologies). However, for these tests, each detection block contains only two ${ }^{3} \mathrm{He}$ detectors, the other cavities being filled with polyethylene cylinders. The sensitive length of the counters is $45 \mathrm{~cm}$, their diameter is $2.5 \mathrm{~cm}$ (1 inch) and their internal ${ }^{3} \mathrm{He}$ pressure is $4 \times 10^{5} \mathrm{~Pa}$ (4 bar).

A 32 channels MEDAS acquisition board from Cesigma dates logic TTL signals from the amplification/discrimination electronics with minimum time bins of $100 \mathrm{~ns}$ [4]. Its clock frequency is $80 \mathrm{MHz}$.

\footnotetext{
*Corresponding author: mehdi.benmosbah@cea.fr.

${ }^{1}$ CEA, DEN, Cadarache, DTN, SMTA, Laboratoire de Mesures Nucléaires, F-13108 Saint-Paul-Lez-Durance, France.

${ }^{2}$ CEA, DEN, Cadarache, DER, SPESI, LDCI, F-13108 Saint-Paul-Lez-Durance, France.

${ }^{3}$ Orano Group, Recycling Business Unit, F-92084 Paris-La Défense, France.
} 
The distance between the two detection blocks is $40 \mathrm{~cm}$. A ${ }^{252} \mathrm{Cf}$ source placed between the two blocks is used to determine their detection efficiency and die-away time.

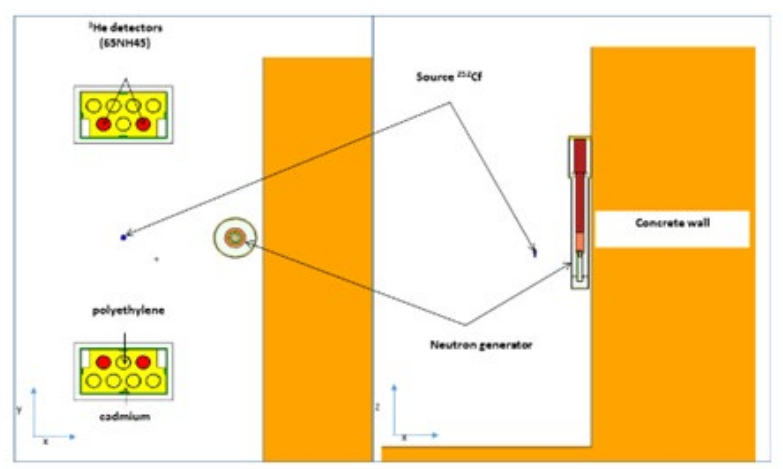

Figure 1: Setup of passive detection tests with a ${ }^{252} \mathrm{Cf}$ source placed in the middle and at mid height of the two detection blocks.

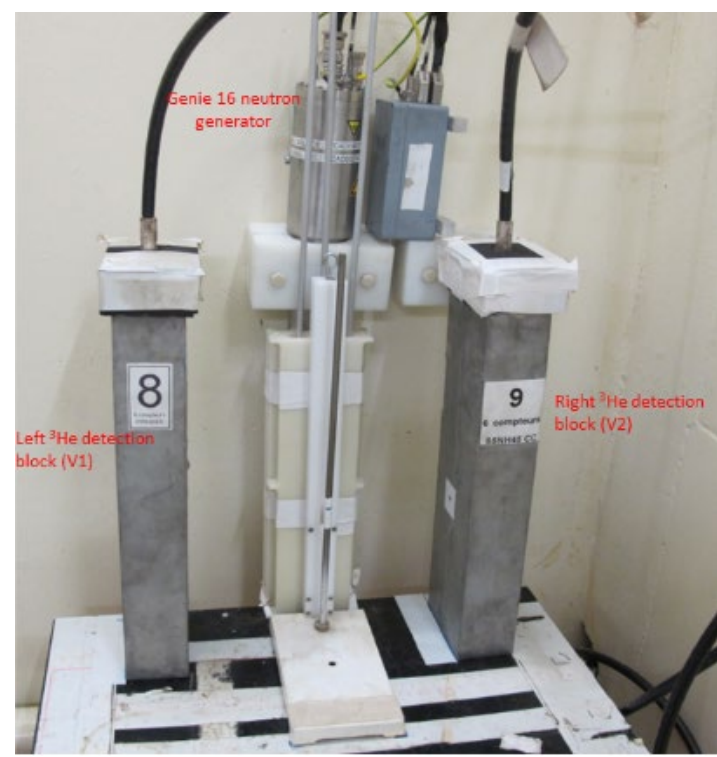

Figure 2: Picture of the experimental setup.

\section{ELECTRONICS SETTINGS}

\section{A. The 7820 current amplifier}

The 7820 current amplifier is combined with 7821 power supply from Mirion Technologies. This amplifier is designed for high-count rate (up to $5.10^{5}$ counts per second) and high gamma dose rates (up to $200 \mathrm{mGv} / \mathrm{h}$ with ${ }^{3} \mathrm{He}$ detectors). In addition, ${ }^{3} \mathrm{He}$ detectors can be connected to such current amplifier with very long cables (up to $100 \mathrm{~m}$ ) while with charge amplifiers short cables are mandatory (maximum $1 \mathrm{~m}$ ).

The connection between the 7820/7821 electronics and the detector is done with a unique coaxial cable with a high immunity to electromagnetic perturbation. The cable is adapted at an impedance of $50 \Omega$ at the input of the amplifier.
The operating High Voltage is $1250 \mathrm{~V}$ and the discrimination threshold is $400 \mathrm{mV}$.

\section{B. PDT electronics}

PDT10A and PDT12S+PDT10M (Figure 3) are compact neutron modules [5] designed for high radiation environments. The PDT10A is directly connected to ${ }^{3} \mathrm{He}$ detectors. The PDT12S module is directly connected to detectors and linked with a cable (30 m maximum length) to PDT10M module. This last allows remote settings of the gain and the discrimination threshold. Each instrument contains a precision wide band charge sensitive pre-amplifier followed by an amplifier, pulse shaping and discriminator circuits. An U8001A Agilent device provides the low voltage $+12 \mathrm{~V}$ power supply of PDT modules. A Cyberstar multichannel power supply provides the High Voltage.

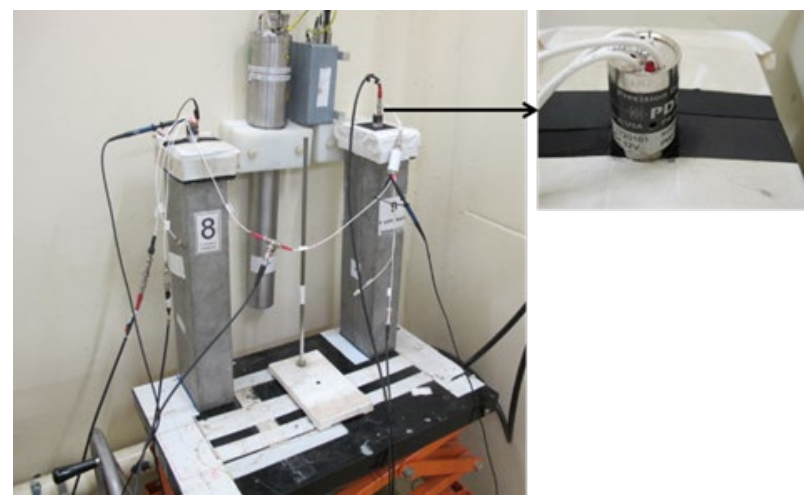

Figure 3 : PDT10A module connected directly to ${ }^{3} \mathrm{He}$ detection block \#8 (on the left), and PDT12S module connected to detection block \#9 (on the right) linked to the PDT10M module. The right picture is a zoom on the PDT12S module.

The PDT modules provide an analog monitoring test point allowing output signal visualization with an oscilloscope, and a TTL pulse used for neutron counting and dating with MEDAS acquisition card.

The operating High Voltage for PDT10A and PDT12S+PDT10M are respectively $1225 \mathrm{~V}$ and $1175 \mathrm{~V}$ and the discrimination threshold is not adjustable however it seems that it is appropriate for the measurements (good discrimination between neutron pulses and noise/gamma pulses, see next sections).

\section{Mesytec electronics}

Both the Mesytec MRS-2000-1 and MRS2000-2 devices (see Figure 4) include a preamplifier, a pulse shaper, a window discriminator and the corresponding TTL output [6]. 
The Mesytec MRS-2000 model is a square shape amplifier with baseline restorer. The experimental tests were carried out with MRS2000-1 designed for very fast counting (pulse FWHM = 1 $\mu$ s for a maximum count rate of $150,000 \mathrm{~s}^{-1}$ indicated by the manufacturer) and with MRS2000-2 designed for standard use (pulse FWHM $=3 \mu \mathrm{s}$ FWHM for a maximum count rate of $\left.50,000 \mathrm{~s}^{-1}\right)$.

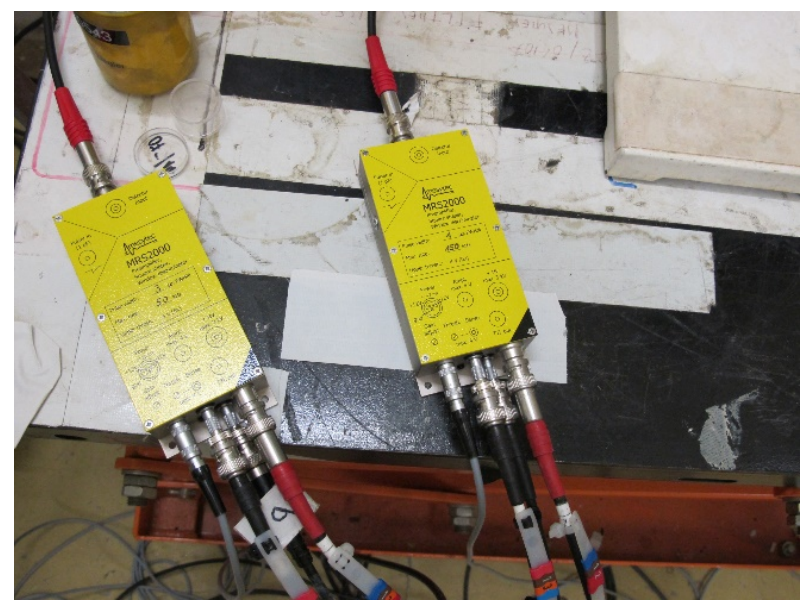

Figure 4 : Mesytec modules (MRS2000-1 on the left and MRS2000-2 on the right).

We set a $1075 \mathrm{~V}$ operating high voltage for both MRS2000-1 and MRS2000-2 modules. The discrimination threshold was set to $100 \mathrm{mV}$.

\section{MONACO electronics}

We tested the performance of a MONACO ("Multichannel Online Neutron Acquisition in Campbell mOde") prototype, which was originally developed for fission chamber measurements in experimental reactors. This electronics works in different modes (pulse, current, Campbell) and we use here the pulse-counting mode.

The MONACO device (see Figure 5) is packed in a 19-inch standard NIM mechanics. Four HN connectors - High Voltage of the Neill connector - allows the connection of up to four detectors. MONACO also provides the High Voltage for the ${ }^{3} \mathrm{He}$ detectors. An analog output enables to display the signal with an oscilloscope. Additionally, a control interface allows adjusting the HV and threshold discrimination values. The reference [3] outlines further details about the MONACO prototype.

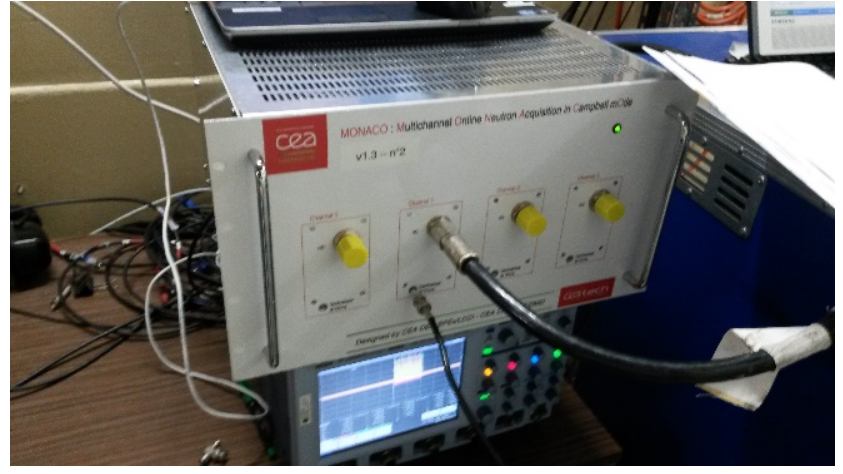

Figure 5 : MONACO prototype electronics.

As MONACO does not provide any TTL output for neutron counting, a Single Channel Analyzer (SCA) was used to convert the analog signal into a TTL pulse that is counted by MEDAS acquisition card. An inverter module also converts the negative analog signal into positive pulses that can be processed by the SCA.

The High Voltage is $1200 \mathrm{~V}$.

Regarding discrimination threshold settings, we use a $10 \mathrm{mV}$ value to eliminate electronics noise. Note that MONACO electronics delivers a very small analog pulse height of less than $50 \mathrm{mV}$ because its gain was not optimized for ${ }^{3} \mathrm{He}$ detectors but for fission chambers. Therefore, discrimination threshold is very small $(10 \mathrm{mV})$ compared to other tested electronics (larger than $100 \mathrm{mV}$ with 7820, Mesytec, and PDT modules).

\section{COMPARATIVE TESTS}

\section{A. Setup and measured parameters}

Measurements are carried out with a ${ }^{252} \mathrm{Cf}$ source placed between the two ${ }^{3} \mathrm{He}$ detection blocks. Its neutron emission rate at the date of measurements is $44893 \pm 823 \mathrm{n} / \mathrm{s}$. We first report here below the main features of the analog output pulse which are: average pulse height, time width (FWHM), overshoot or undershoot, pulse height distribution and discrimination threshold position with respect to electronic noise and gamma background, on the one hand, and neutron pulses on the other hand. Then we present the main parameters for total and coincidence counting [7], i.e. neutron detection efficiency and die-away time in the detection blocks.

\section{B. ACH-NA98 charge amplifier}

The ACH-NA98 charge amplifier, which is the reference for neutron detection efficiency in this work, was not calibrated and tested in the same conditions because all its characteristics (pulse amplitude and width, Rossi- $\alpha$ curve, etc.) were already available. They were obtained from previous measurements [8] and are reported below (see Table I). Only its detection efficiency was determined in this work, for further comparisons. 
TABLE I : CHARACTERISTICS OF ACH-NA98 CHARGE AMPLIFIER

\begin{tabular}{ll} 
Parameter & Value \\
\hline Operating High Voltage & $1400 \mathrm{~V}$ \\
Discrimination threshold & $200 \mathrm{mV}$ \\
Pulse FWHM & $1 \mu \mathrm{s}$ \\
Mean pulse amplitude & $1 \mathrm{~V}$ \\
Undershoot & $200 \mathrm{mV}$ \\
Die-Away Half Time & $32 \mu \mathrm{s}$ \\
Detection efficiency & 1 (reference device) \\
\hline
\end{tabular}

In the following section, we present detailed results for the PDT devices only. For other devices, results are summarized in section $\mathrm{V}$.

\section{PDT electronics}

The oscilloscope screenshot of Figure 6 shows the output signals of the PDT10A module. The average pulse height of the analog signal is $161 \mathrm{mV}$ and its width (FWHM) is $1.41 \mu \mathrm{s}$, to be compared to the FWHM of 350 ns obtained with the $7820 / 7821$ electronics. Note that these average values for PDT10A, have been determined for 1226 pulses.



Figure 6 : Average analog signal (green; $50 \mathrm{mV} /$ div) and logical TTL signal (pink; $2 \mathrm{~V} /$ div) for the PDT10A module (time base $500 \mathrm{~ns} /$ div).

As observed in Figure 7, the analog pulse has a noticeable undershoot of about $20 \mathrm{mV}$ and returns to the baseline only after $45 \mu \mathrm{s}$. This undershoot can induce count losses because another pulse arriving in this time interval might mistakenly be rejected by the low discrimination threshold due to its smaller apparent height.

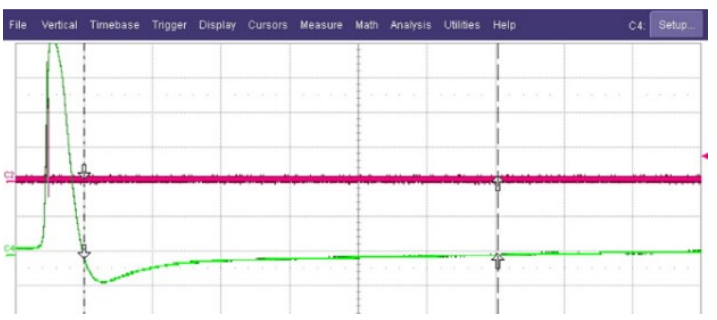

Figure $7:$ Zoom on the overshoot observed for the average analog pulse (green; $20 \mathrm{mV} / \mathrm{div}$ ) of the PDT10A module (time base $5 \mu \mathrm{s} / \mathrm{div}$ ).

Figure 8 presents the pulse height distribution of the analog signal of the PDT10A module, which varies from 65 to $375 \mathrm{mV}$. This Figure illustrates that the discrimination threshold (corresponding to the factory value for ${ }^{3} \mathrm{He}$ detectors) allows removing the electronic noise.

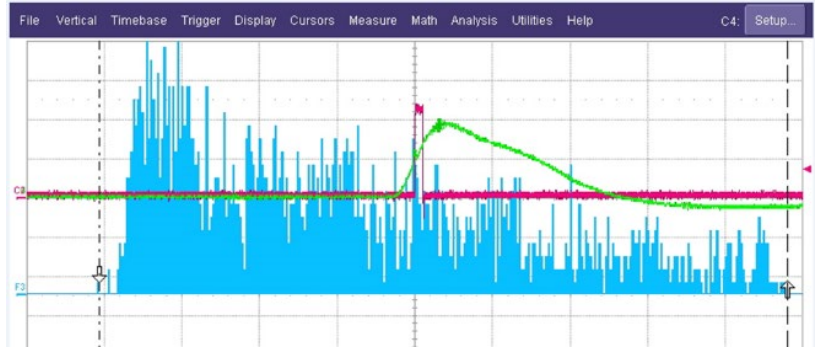

Figure 8 : Pulse height distribution of the analog signal for the PDT10A module starting at $65 \mathrm{mV}$ ( 3 events/div; $35 \mathrm{mV} /$ div for the horizontal scale).

Figure 9 displays the average analog pulse of the PDT12S+PDT10M module for 1197 events. The average pulse height is $190 \mathrm{mV}$ and its average FWHM is $1.6 \mu \mathrm{s}$.

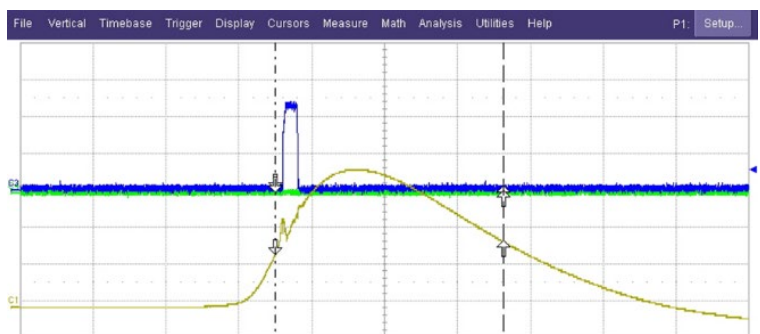

Figure 9 : Average analog signal (yellow; $50 \mathrm{mV} / \mathrm{channel)}$ and logical TTL signal (blue; 2V/channel) for the PDT12S+PDT10M module (time base 500 ns/channel).

Figure 10 shows that the PDT12S+PDT10M pulse also exhibits an undershoot of $50 \mathrm{mV}$ and returns to the baseline only after $60 \mu \mathrm{s}$.

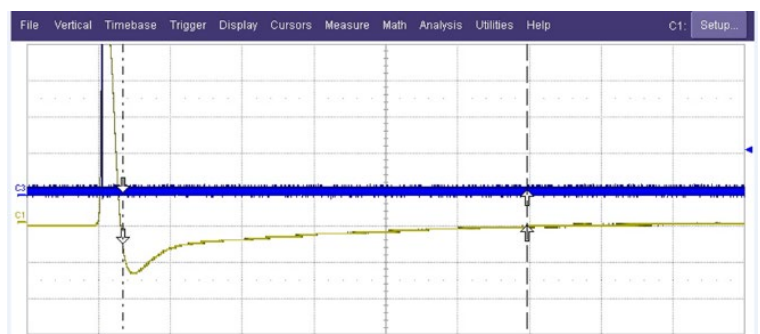

Figure 10: Zoom on the undershoot observed for the analog pulse of the PDT12S+PDT10M module (20 mV/channel; time base $10 \mu \mathrm{s} / \mathrm{channel})$.

The pulse height distribution, shown in Figure 11 varies from $67 \mathrm{mV}$ to $549 \mathrm{mV}$. As observed for the PDT10A module, the pulse height distribution seems to indicate that the discrimination threshold (corresponding to the factory value, which is not specified by the manufacturer) to cut the electronic noise. 


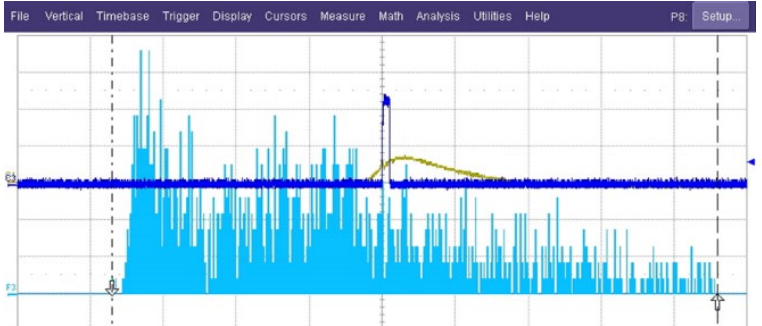

Figure 11 : Pulse height distribution of the analog signal for the PDT12S+PDT10M module starting at $67 \mathrm{mV}$ (2.25 events/channel ; 44 $\mathrm{mV} /$ div).

In conclusion, as PDT charge amplifiers deliver much longer pulses $(\sim 1.4$ to $1.6 \mu \mathrm{s}$ FWHM) than 7820 current amplifier $(80$ ns FWHM), they are more sensitive to pile-up effects at high neutron or gamma count rates.

The experimental Rossi- $\alpha$ curves obtained with a ${ }^{252} \mathrm{Cf}$ source are presented in Figure 12. The die-away half times are obtained with the fit of a decreasing exponential function: $35.98 \pm 1.18$ $\mu \mathrm{s}[1 \sigma]$ for PDT10A module and $35.10 \pm 1.15 \mu \mathrm{s}[1 \sigma]$ for PDT12S+PTD10M module. These values of the same order of magnitude as the Die Away Half Time measured for the 7820 amplifier: $45.1 \pm 5.5 \mu \mathrm{s}[1 \sigma]$.

(a)

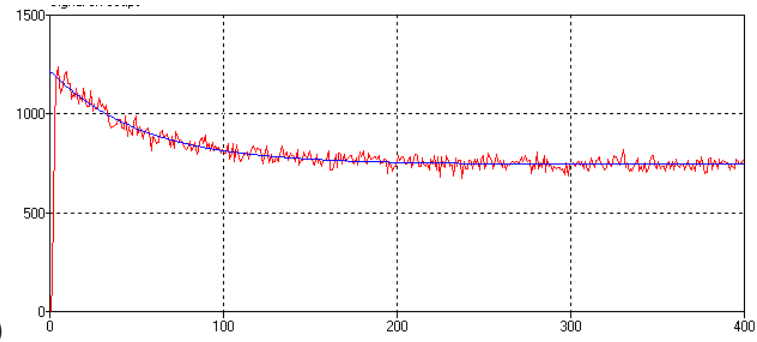

(b)



Figure 12 : Rossi- $\alpha$ curves (histogram of pulse arrival times after a first detection, in $\mu \mathrm{s}$ ): (experimental in red; adjustment in blue) for PDT10A (a) and PDT12S+PTD10M (b).

We finally determine the experimental detection efficiency reported in with the ${ }^{252} \mathrm{Cf}$ neutron source. For PDT12S+PDT10M module, it is similar to the reference efficiency obtained with ACH-NA98 charge amplifier, which provides a very good signal-to-noise ratio and therefore small neutron count losses due to electronics threshold. In contrast, it is not the case for the PDT10A device, which displays a detection efficiency that is $30 \%$ lower (ratio vs. ACH-NA98 of $0.7)$.
TABLE II : PDT vs. ACH-NA98 detection efficiencies. the measured data of ACH-NA98 are available in table II.

\begin{tabular}{llc} 
Parameter & PDT10A & PDT12S+PDT10M \\
\hline $\begin{array}{l}\text { Measured } \\
\text { count rate }(\mathrm{c} / \mathrm{s})\end{array}$ & $171.59 \pm 0.18$ & $258.4 \pm 0.21$ \\
$\begin{array}{l}\text { Detection } \\
\text { efficiency }\end{array}$ & $0.38 \pm 0.01$ & $0.57 \pm 0.01$ \\
$\begin{array}{l}\text { Ratio } \\
\text { vs. ACH-NA98 }\end{array}$ & 0.7 & 1 \\
\hline
\end{tabular}

\section{SUMmary}

Table III summaries the main characteristics of the amplification/discrimination electronics tested in this paper.

TABLE III: COMPARISON OF MAIN CHARACTERISTICS OF TESTED ELECTRONICS.

\begin{tabular}{cccccc}
\hline Device & $\begin{array}{c}\text { Pulse } \\
\text { FWHM } \\
(\mu \mathrm{s})\end{array}$ & $\begin{array}{c}\text { Pulse } \\
\text { Amplitude } \\
(\mathrm{mV})\end{array}$ & $\begin{array}{c}\text { Undershoot } \\
(\mathrm{mV})\end{array}$ & $\begin{array}{c}\text { Die } \\
\text { Away } \\
\text { Half } \\
\text { Time } \\
(\mu \mathrm{s})\end{array}$ & $\begin{array}{c}\text { Detection } \\
\text { Efficiency } \\
\text { Ratio } \\
(\text { ACH- } \\
\text { NA98 }=1)\end{array}$ \\
7820 amplifier & 0.35 & 1177 & 100 & 33 & 0.7 \\
ACH-NA98 & 1 & 1000 & 200 & 32 & 1 \\
PDT10A & 1.41 & 161 & 20 & 35.98 & $\approx 1$ \\
PDT12S+ & 1.57 & 186 & 50 & 35.1 & $\approx 1$ \\
PDT10M & & & & & \\
MRS2000-1 & 1.55 & 1531 & 5 & 37 & $\approx 1$ \\
MRS2000-2 & 3.44 & 658 & 10 & 40 & $\approx 1$ \\
MONACO & 1.5 & 69 & none & 35.4 & 0.7 \\
\hline
\end{tabular}

The time width (FWHM) of the output pulse of the PDT10A, PDT12S+PDT10M, MRS2000-1 and MONACO devices is similar, around $1.5 \mu \mathrm{s}$. The FWHM of the 7820 current amplifier pulse is much shorter (350 ns) and therefore, it can be operated at high count rate (up to $5 \times 10^{5} \mathrm{n} / \mathrm{s}$ [7]). Additionally, thanks to limited pulse pile-up, it can be operated at high gamma count rate (up to a $200 \mathrm{mGy} \cdot \mathrm{h}^{-1}$ gamma dose rate [7]; note that neither high count rate, nor high gamma level tests have been performed in this work). Finally, the MRS2000-2 analog pulse FWHM is the longest $(3.4 \mu \mathrm{s})$ of all tested devices and would leads to the largest signal processing dead time.

Detection efficiencies of all tested electronics have been compared to that of the reference ACH-NA98 charge amplifier. The PDT10A, PDT12S+PDT10M, MRS-2000-1 and MRS2000-2 modules give similar efficiencies as ACH-NA98, whereas MONACO is comparable to 7820 current amplifier with about $30 \%$ count losses.

Finally, the neutron die-away half time in the detection blocks varies between 35 and $45 \mu$ s for all tested electronic devices, which shows that all these electronics can be used for passive neutron coincidence counting.

\section{CONCLUSION}

In the field of a technology survey, five electronics have been tested for passive neutron counting with ${ }^{3} \mathrm{He}$ gas proportional counters and compared to 7820 current amplifier and ACHNA98 charge amplifier, both from Mirion technologies, which are commonly used at Nuclear Measurement Laboratory of CEA, DEN, Cadarache. The new tested electronics are the 
PDT10A and PDT12S+PDT10M modules from Proportional Technologies, the MRS2000-1 and MRS2000-2 modules from Mesytech, and finally the MONACO prototype electronics from CEA LIST.

The PDT and Mesytech devices lead to an equivalent detection efficiency to that of ACH-NA98 charge amplifier, which allows the best signal-to-noise ratio. However, detection efficiency obtained with MONACO is smaller, equivalent to that of Mirion 7820 current amplifier designed for high-count rate measurements with very short pulses to limit pile-up. Note that MONACO is designed for fission chamber measurements and its settings need to be adapted to ${ }^{3} \mathrm{He}$ counters.

Analog signals of all tested electronics display pulse widths of a few $\mu$ s (FWHM), which is much longer than the 7820 current amplifier (350 ns), but without noticeable influence on neutron die-away time in the detections blocks made of polyethylene and cadmium surrounding ${ }^{3} \mathrm{He}$ counters. Consequently, all these electronics can be used for passive neutron coincidence counting with ${ }^{3} \mathrm{He}$ detectors at low and intermediate count rates. However, Mirion 7820 is recommended for high count rate applications, to limit pulse pile-up and improve gamma rejection.

This series of comparative tests will be continued in active mode with a DT pulsed neutron generator, in view of active neutron interrogation applications with the Differential DieAway Technique [9] [10].

\section{REFERENCES}

[1] G.F. Knoll, Radiation Detection and Measurement. New York, John Wiley and Sons, 1999.

[2] A. Boffa, A. Fazzi, C. Pirovani and V. Varoli. "A single-supply, high rate, small size and cheap electronic chain for $3 \mathrm{He}$ neutron counters". Nuclear Science Symposium, 1996, IEEE Conference Record.

[3] M. Thevenin, L. Barbot, G. Corre, R. Woo, C. Destouches and S Normand. "Digital Real-Time Multiple Channel Multiple Mode Neutron Flux Estimation on FPGA-based Device". Conference Paper in The European Physical Journal Conferences. May 2014.

[4] Cesigma Signal and Systems. [Online] Available: http://www.cesigma.net/ang/carte-medas-A.html

[5] Precision Data technology. "PDT10A-HN General Specifications". http://www.pdt-inc.com/products/10series/10series_datasheet.htm

[6] Mesytec. "MRS2000 data sheet Version 1.2 01". [Online] Available: http://www.mesytec.de/products/datasheets/MRS-2000.pdf

[7] B. Pérot, F. Jallu, C. Passard, O. Gueton, PG. Allinei, L. Loubet, N. Estre E. Simon, C. Carasco, C. Roure, L. Boucher, H. Lamotte, J. Comte, M. Bertaux, P. Fichet, F. Carrel, A. Lyoussi, "The characterization of radioactive waste: a critical review of techniques implemented or under development at CEA, France", EPJ Nuclear Sci. Technol. 4, 3 (2018) 1 24

[8] C. Passard. CEA Technical Report, unpublished. 2015.

[9] R. Antoni, C. Passard, B. Perot, F. Guillaumin, C. Mazy, M. Batifol, G. Grassi, Reduction of the uncertainty due to fissile clusters in radioactive waste characterization with the Differential Die-away Technique, Nuclear Inst. and Methods in Physics Research, A 895 (2018) 144-149

[10] R. Antoni, C. Passard, B. Perot, M. Batifol, J-C. Vandamme, G. Grassi, First Industrial Tests of a Matrix Monitor Correction for the Differential Die-away Technique of Historic Waste Drums, ANIMMA 2015, Advancements in Nuclear Instrumentation Measurement Methods and their Applications, 20-24 April 2015, Lisbon, Portugal. 